\title{
Transplantation of bone mesenchymal stem cells promotes angiogenesis and improves neurological function after traumatic brain injury in mouse
}

This article was published in the following Dove Press journal:

Neuropsychiatric Disease and Treatment

6 November 2017

Number of times this article has been viewed

\section{Shewei Guo* \\ Yingwei Zhen* \\ Anran Wang}

Department of Neurosurgery, The First Affiliated Hospital, Zhengzhou University, Henan, China

*These authors contributed equally to this work
Correspondence: Shewei Guo

Department of Neurosurgery, The First Affiliated Hospital, Zhengzhou University, No I Jianshe East Road, Henan, 450000, China

Tel +86 I88 38065390

Fax +86 37| 669| 3||4

Email gswfeicui@sohu.com
Abstract: Traumatic brain injury (TBI) has emerged as a leading cause of mortality and morbidity worldwide. Transplantation of bone mesenchymal stem cells (BMSCs) has emerged as a promising treatment for various central nervous system diseases. This study aims to evaluate the effect of BMSCs transplantation by intravenous injection on neurological function and angiogenesis of the TBI mice. C57BL/6 male mice were randomly divided into four groups: control, sham, TBI, and BMSC. Functional neurological evaluation was performed 1, 4, 7, 14, and 21 days after TBI using neurological severity scores. The impairment of learning and memory in mice was evaluated 14 days after TBI by Morris water maze experiment. Mice were sacrificed 14 days after TBI, and then brain sections were stained by terminal deoxyribonucleotidyl transferase (TDT)-mediated dUTP-digoxigenin nick end labeling staining to assess brain neuronal apoptosis. Immunohistochemistry was conducted to evaluate caspase-3 activity and identify vascular distribution and microvessel density. Protein and mRNA levels of vascular endothelial growth factor (VEGF) and angiogenin-1 (Ang-1) in brain tissues were analyzed by Western blot and quantitative real-time polymerase chain reaction, respectively. BMSCs transplantation promoted recovery of neurological function, ameliorated impairment of learning and memory, attenuated neuronal apoptosis, and diminished caspase- 3 activation in mice after TBI. Also, BMSCs transplantation upregulated expressions of VEGF and Ang-1 and promoted the formation of microvessels in brain tissues after TBI. Our study demonstrated the important role of BMSCs transplantation in TBI mouse and indicated that the underlying mechanism was through promoting angiogenesis and improving neurological function. This provides a novel and effective strategy for cell-based therapy in the treatment of TBI.

Keywords: TBI, BMSCs, angiogenesis, neurological function

\section{Introduction}

Traumatic brain injury (TBI) has emerged as a leading cause of mortality and morbidity worldwide. ${ }^{1}$ It is defined as an alteration in brain function and/or other evidence of brain pathology caused by a sudden external mechanical force, such as rapid acceleration or deceleration, blast waves, crush, an impact, or penetration by a projectile. ${ }^{1,2}$ TBI survivors frequently suffer from temporary or permanent impairment of cognitive, physical, and psychosocial functions. ${ }^{3-5}$ It is widely acknowledged that the neurons in the human hippocampus, which are critical for learning and memory, ${ }^{6}$ are extremely vulnerable to brain trauma. Once injured, the neurons will experience massive degeneration and necrosis, leading to dysfunction of learning and memory. Despite major progress in understanding the pathophysiology of TBI, promising neuroprotective drugs effective in animal TBI models, unfortunately, have all failed 
in Phase II or Phase III clinical trials. ${ }^{1}$ Therefore, further investigation in pharmacological treatments, neural repair and regeneration, or other treatments is urgently needed to improve outcomes for TBI patients. ${ }^{7}$

Bone mesenchymal stem cells(BMSCs) provide hematopoietic support and exhibit immunoregulation function, with multilineage differentiation potential (pluripotency) and self-renewal ability. ${ }^{8}$ The transplanted BMSCs could be differentiated into neurons and glial cells. ${ }^{9}$ Of note, BMSCs have been shown to be an excellent candidate for cell therapy. ${ }^{10}$ They can be obtained from autologous donation, thus eliminating ethical disputes and other concerns associated with graft rejection. ${ }^{11}$ In addition, clinical studies have shown that human BMSCs would not cause the activation of the host's immune system, ${ }^{12}$ suggesting that even allogeneic BMSCs can be used for transplantation therapy. Human trials have shown so far no adverse effects on allogeneic vs autologous BMSCs transplantation. ${ }^{13}$ Transplantation of BMSCs has been shown to promote the repair and regeneration of nerve tissues in the central nervous system (CNS) ${ }^{14}$ and has emerged as a promising treatment for various CNS diseases, ${ }^{15}$ such as stroke, TBI, intracerebral hemorrhage, and spinal cord injury.

In our study, BMSCs were transplanted into TBI model to evaluate the effect of BMSCs on neurological function and angiogenesis of the host mice. Also, the related underlying mechanism was further explored. Our study provided supports for the clinical application of BMSCs in the treatment of TBI.

\section{Materials and methods BMSCs culture}

BMSCs were harvested as previously described by Wei et al. ${ }^{16}$ In brief, mice were anesthetized and sacrificed by cervical dislocation. BMSCs were rinsed out from the femurs and tibias of mice by a 20-gauge syringe. The cells were suspended in DMEM containing 10\% fetal bovine serum and incubated at $37^{\circ} \mathrm{C}$ with $5 \% \mathrm{CO}_{2}$ for 24 hours. The medium containing the nonadherent cells was removed, and then fresh medium was added to allow for selection by plastic adhesion. After BMSCs isolation, the medium was replaced every 3 days, and the cultures were passaged when the BMSCs reached $80 \%$ confluence. To confirm cell characteristics of cultured cells, BMSCs were subjected to fluorescence-activated cell sorting using CD90, CD34, and CD45 markers, and BMSCs were identified as CD90 positive and CD34/CD45 negative cells.

\section{Animals}

C57BL/6 male mice weighing 20-25 g were purchased from Laboratory Animal Center of Shanghai Institutes for Biological Science. This study was approved by the Ethics Committee of the First Affiliated Hospital of Zhengzhou University. All animal procedures were in compliance with the National Institutes of Health (NIH) Guidelines for the Care and Use of Laboratory Animals. Mice ( $\mathrm{N}=60)$ were randomly divided into four groups with 15 for each: control, sham (received sham operation without cortical impact), TBI (received TBI injury), and BMSC (received intravenous injection of $2 \times 10^{6} / \mu \mathrm{L}$ BMSCs at tail vein after TBI).

\section{Establishment of mice TBI model}

Establishment of mice TBI model was performed as described by Hunt et al, ${ }^{17}$ with some alterations. Briefly, mice were anesthetized with $4 \%$ chloral hydrate $(0.4 \mathrm{mg} / \mathrm{g})$ and positioned in a stereotaxic apparatus. A midline incision was made to expose the skull. A 4-mm craniotomy was performed lateral to the sagittal suture and centered between bregma and lambda using a dental drill. The skull cap was carefully removed without disruption of the exposed underlying dura, and mice were then subjected to TBI using a controlled cortical impact device. A standardized parietal contusion was performed by letting a $20 \mathrm{mg}$ steel rod with a flat end and a diameter of $2 \mathrm{~mm}$ drop on a piston resting on the dura from a height of $50 \mathrm{~cm}$. The piston was allowed to compress the cortex to a depth of $1.0 \mathrm{~mm}$, with the same duration. After injury, the incision was sutured, and mice were carefully observed and nursed. Each group guaranteed at least five animals for the final surviving behavior, water maze, and morphological examination.

\section{Neurological assessment}

A standardized neurological severity score (NSS) was performed to assess the extent of neurological impairment in a blinded fashion by two different investigators at defined time intervals after trauma $(\mathrm{t}=1,4,7,14$, and 21 days), as described by Leinhase et al and Stahel et al. ${ }^{18,19} \mathrm{NSS}$ consists of 10 individual parameters, including tasks on motor function, alertness, and physiological behavior, where one point indicates failure of the task and no point indicates success. The maximum NSS score (10 points) reflects the severest neurological impairment with failure of all tasks.

\section{Morris water maze}

To evaluate the impairment of learning and memory in mice, Morris water maze (MWM) experiment was performed 
14 days after TBI experiment, as described previously ${ }^{20,21}$ with some alterations. The apparatus includes a circular pool (180 $\mathrm{cm}$ in diameter, $45 \mathrm{~cm}$ in depth) filled with water (30 $\mathrm{cm}$ in depth) and plexiglass platform $(10 \mathrm{~cm}$ in diameter). The pool is virtually subdivided into four equal quadrants. The platform was placed $1 \mathrm{~cm}$ beneath the surface of the water. Animal behaviors were monitored by the EthoVision system. Mice which were able to find the platform within 20-40 seconds in the training session were selected for MWM experiment. Spatial navigation (invisible platform) and probe trials were performed. In brief, the platform was placed at the center of a fixed quadrant. The mice were placed in the water at the opposite side of the platform and the time they spent reaching the underwater platform is the escape latency. After the completion of spatial navigation trial, original platform was removed and the mice were placed in the water at opposite site of the original platform, and the swimming tracks of mice within 2 minutes were recorded. The percentage of target zone entries, the percentage of time spent finding the target zone, and the number of times crossing the site (original platform) were calculated.

\section{Neuronal apoptosis assay and evaluation of caspase- 3 activity}

Fourteen days after TBI experiment, four mice in each group were randomly selected and sacrificed under anesthesia. Brain tissues were isolated and paraffin sections were prepared. To evaluate the apoptosis of brain neurons, the sections were subjected to terminal deoxyribonucleotidyl transferase (TDT)-mediated dUTP-digoxigenin nick end labeling (TUNEL) staining. Briefly, the sections $(4 \mu \mathrm{m})$ were dewaxed, hydrated, and washed. After that, proteinase $\mathrm{K}$ $(20 \mu \mathrm{L} / \mathrm{mL})$ and Triton $\mathrm{X}-100$ in $0.1 \%$ sodium citrate were added successively. Then the sections were incubated in $0.3 \%$ $\mathrm{H}_{2} \mathrm{O}_{2}$ /methanol, followed by incubation with TUNEL reaction mixture (50 g; Boehringer Co., Mannheim, Germany) at $37^{\circ} \mathrm{C}$ in a humidified chamber. Finally, the sections were stained with diaminobenzidine (DAB). For evaluation of caspase-3 activity, immunohistochemical staining was conducted to observe the expression of caspase-3. Images were acquired by an IX81 Olympus microscope (Olympus, Tokyo, Japan).

\section{Western blot}

Fourteen days after TBI experiment, four mice in each group were randomly selected and sacrificed under anesthesia. Brain tissues were isolated and subjected to Western blot. In brief, tissues were lysed in in $200 \mu \mathrm{L}$ lysis buffer. Then equal amount of protein from cell lysates was separated by
$10 \%$ sodium dodecyl sulfate-polyacrylamide gel electrophoresis gels and transferred to polyvinylidene difluoride membrane (EMD Millipore, Billerica, MA, USA). After that, primary antibodies against vascular endothelial growth factor (VEGF) or angiogenin-1 (Ang-1) (Santa Cruz Biotechnology Inc., Dallas, TX, USA) were added, followed by horseradish peroxidase (HRP)-conjugated goat anti-rabbit IgG secondary antibodies. GAPDH was used as an internal control. The protein analysis was visualized by Quantity One software (Bio-Rad Laboratories Inc., Hercules, CA, USA).

\section{RNA extraction and quantitative real-time polymerase chain reaction}

Fourteen days after TBI experiment, four mice in each group were randomly selected and sacrificed under anesthesia. Brain tissues were isolated and subjected to Western blot. Total RNA was extracted from tissues and cells by using Trizol reagent (Thermo Fisher Scientific, Waltham, MA, USA) and was reverse transcribed into cDNAs using the reverse transcription kit (Takara, Shiga, Japan). The cDNA template was synthesized through quantitative real-time polymerase chain reaction (qRT-PCR) using SYBR Premix Dimmer Eraser kit (Takara) by the ABI7900 system (Thermo Fisher Scientific). The relative expressions of VEGF and Ang-1 were calculated by the $2^{-\Delta \Delta C t}$ method and normalized to the internal control GAPDH.

\section{Immunohistochemistry and microvessel counting}

Vascular distribution and microvessel density (MVD) in mice brain tissues was identified immunohistochemically by monoclonal antibodies against CD34, as previously described. ${ }^{22}$ Briefly, paraffin-embedded brain tissues $(4 \mu \mathrm{m})$ were dewaxed, hydrated, and washed. Then $0.3 \% \mathrm{H}_{2} \mathrm{O}_{2}$ was added to quench the endogenous peroxidase activity. After blocking nonspecific staining by incubation with $5 \%$ bovine serum albumin, sections were incubated with the primary specific biotinylated antibodies against CD34 (Cell signaling Technology, Danvers, MA, USA), followed by incubation with HRP-conjugated streptavidin. Staining was visualized with $\mathrm{DAB}$, and sections were counterstained with Mayer's hematoxylin. Images were acquired by a fluorescence microscope. Quantification of MVD was conducted as described by Weidner et al. ${ }^{23}$ The mean value of microvessel counts from five randomly selected fields was recorded as MVD of the section.

\section{Statistical analysis}

All statistical analyses were performed using SPSS 18.0. Differences between two groups were evaluated by Student's 
$t$-test. Data are presented as the mean \pm SD from three independent experiments. Values of $P<0.05$ were considered statistically significant.

\section{Results}

BMSCs transplantation promoted recovery of neurological function in mice after TBI

A standardized NSS was performed to assess the extent of neurological impairment in a blinded fashion at defined time intervals after TBI. The control and sham mice showed relatively normal behavior, as reflected by the low NSS scores. In contrast, a significantly increased NSS was observed at all time intervals after trauma in the TBI mice. However, after treated by transplantation of BMSCs, mice displayed markedly reduced NSS scores (Figure 1). These results implied that transplantation of BMSCs promoted recovery of neurological function.

\section{BMSCs transplantation ameliorated the impairment of learning and memory in mice after TBI}

To evaluate the impairment of learning and memory in mice, MWM experiment was performed 14 days after TBI experiment. For spatial navigation trial, TBI mice greatly prolonged the escape latency, whereas BMSCs-transplanted mice showed reduced latency, close to the level in control and sham groups (Figure 2A). For spatial probe trial, compared to control and sham groups, a reduction in target zone entries,

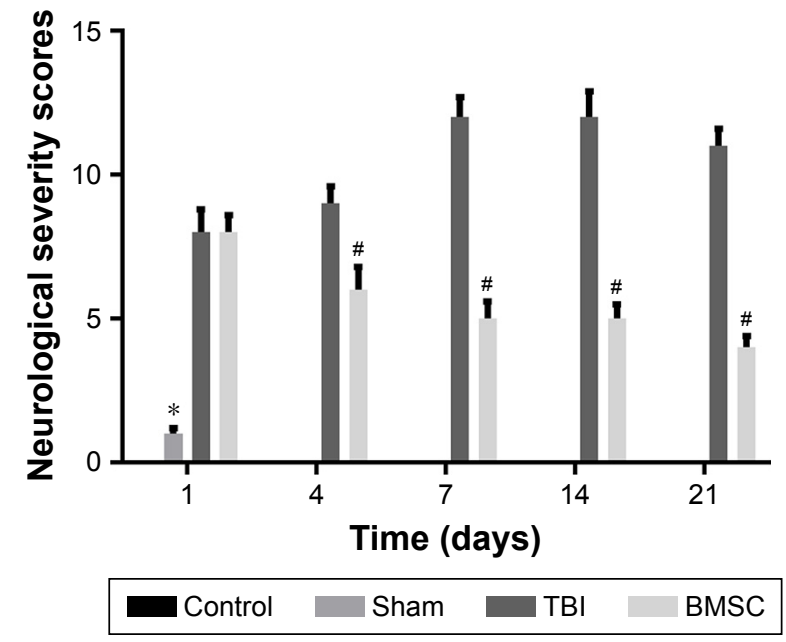

Figure I BMSCs transplantation promoted recovery of neurological function in mice after TBI. The extent of neurological impairment in control, sham, TBI, and BMSC groups was assessed by a standardized neurological severity score (NSS) at defined time intervals after trauma $(\mathrm{t}=1,4,7,14$, and 21 days). $* P<0.05$ : Sham vs TBI, ${ }^{\#} P<0.05$ : BMSC vs TBI.

Abbreviations: BMSC, bone mesenchymal stem cell; TBI, traumatic brain injury. time in target zone, and number of times crossing the site was observed in TBI mice. However, transplantation of BMSCs induced an increase in the previously mentioned parameters (Figure 2B-D). Our results indicated that BMSCs transplantation attenuated learning and memory impairment in mice after TBI.

\section{BMSCs transplantation attenuated neuronal apoptosis and diminished caspase- 3 activation}

Fourteen days after TBI experiment, four mice in each group were randomly selected and sacrificed under anesthesia. Brain tissues were isolated and paraffin sections were prepared. To evaluate the apoptosis of brain neurons, the sections were subjected to TUNEL staining. Data revealed that, compared to control and sham groups, increased TUNELpositive cells exhibiting typical apoptotic morphology including shrunken nuclei, condensed chromatin, and apoptotic bodies were observed in TBI mice. Meanwhile, mice treated with BMSCs transplantation showed reduced TUNEL-positive cells (Figure 3A). Furthermore, caspase-3 expression was evaluated to confirm the ongoing apoptosis (via the extrinsic pathway) by cleaved caspase-3 immunohistochemical staining. Data demonstrated that, compared to TBI group, the activity of caspase-3 was reduced in BMSCs-transplanted mice (Figure 3B). All these results suggested that BMSCs transplantation reduced apoptotic damage induced by TBI in mice.

\section{BMSCs transplantation upregulated expressions of VEGF and Ang-I in brain tissues after TBI}

Brain tissues from randomly selected mice in each group were isolated 14 days after TBI experiment. To evaluate the effect of BMSCs transplantation on angiogenesis of TBItreated mice, expressions of VEGF and Ang- 1 at mRNA and protein levels in brain tissues were analyzed by qRT-PCR and Western blot, respectively. Data revealed that TBI mice showed reduced levels of VEGF and Ang-1 compared to control and sham groups. Interestingly, we found that BMSCs transplantation upregulated VEGF and Ang-1 to levels close to those found in control or sham group (Figure 4).

\section{BMSCs transplantation promoted the formation of microvessels in brain tissues after TBI}

MVD is regarded as a surrogate marker for angiogenesis. ${ }^{22}$ To further evaluate the effect of BMSCs transplantation on 


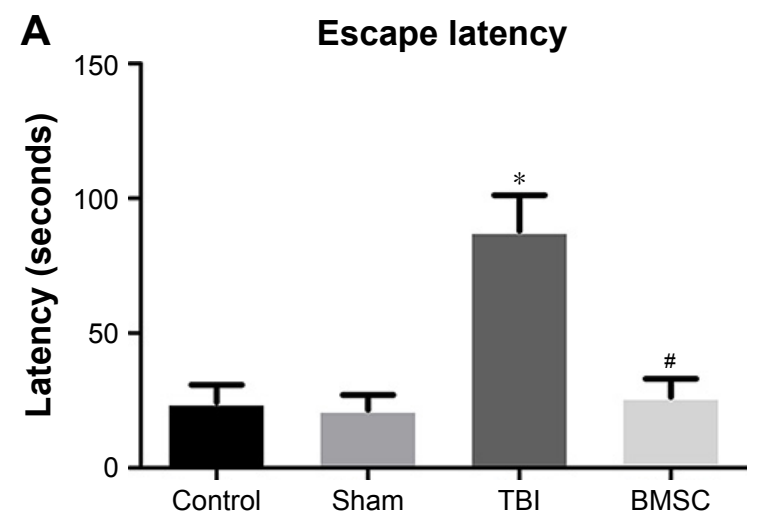

B
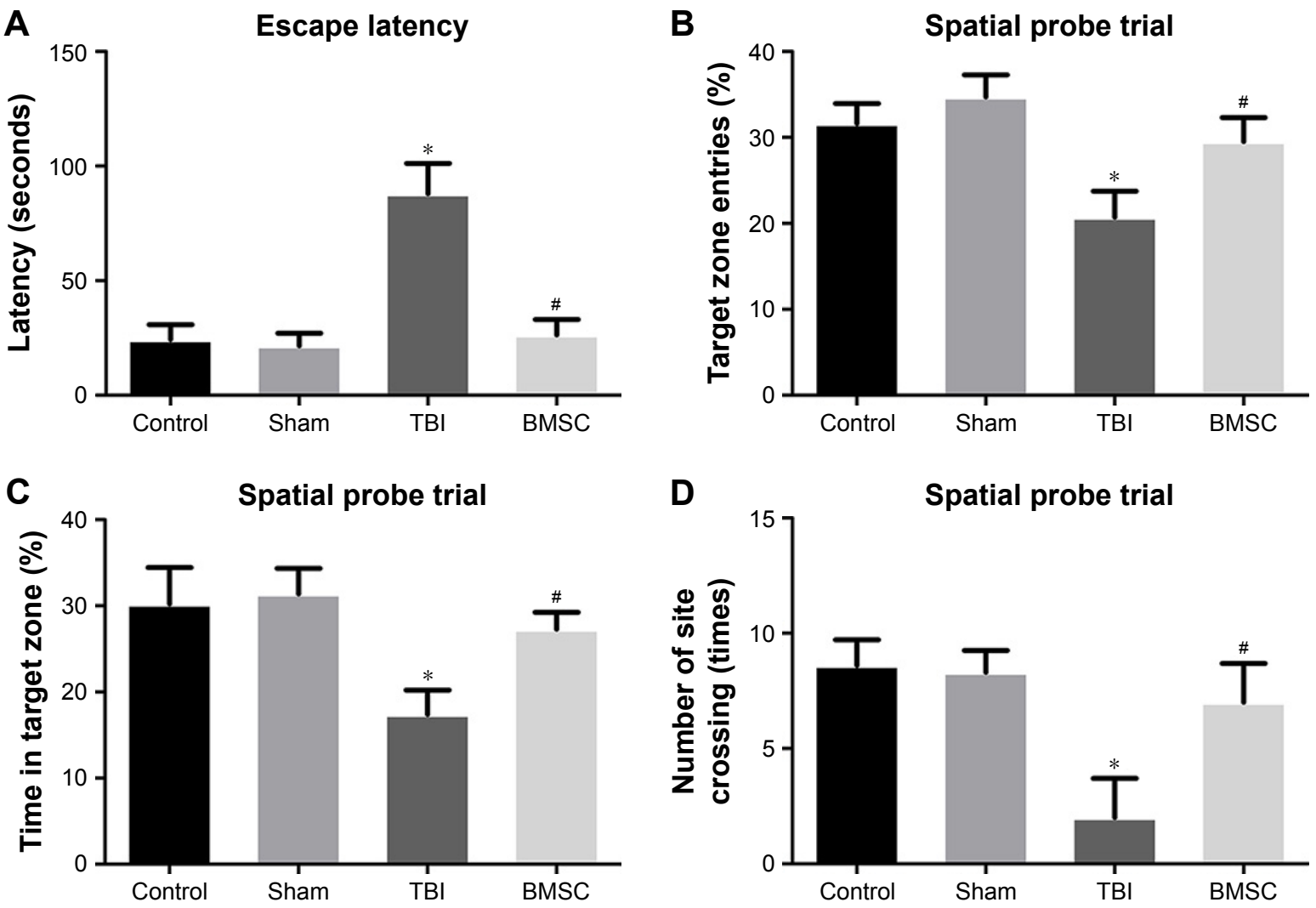

Figure 2 Spatial navigation and probe trials in Morris water maze. (A) The time of latency, (B) the percentage of target zone entries, (C) the percentage of time spent finding the target zone, and (D) the number of times crossing the site (original platform) were calculated. $* P<0.05$ : Sham vs TBI, $\# P<0.05$ : BMSC vs TBI. Abbreviations: BMSC, bone mesenchymal stem cell; TBI, traumatic brain injury.

the formation of microvessels of TBI-treated mice, brain tissues from randomly selected mice in each group were isolated 14 days after TBI experiment, then vascular distribution and MVD in brain tissues was identified immunohistochemically by using monoclonal antibodies against CD34. Data revealed that microvessels within the brain tissues of control and sham mice were strongly stained by CD34. However, microvessels in the brain tissues of TBI mice expressed a minimal level of CD34. Interestingly, mice receiving BMSCs transplantation showed more microvessels stained by $\mathrm{CD} 34$
A

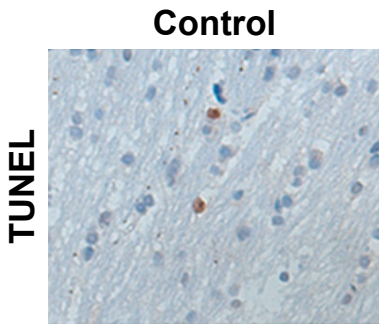

B

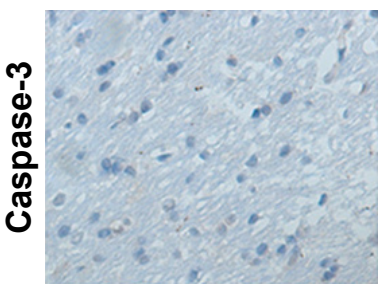

Sham
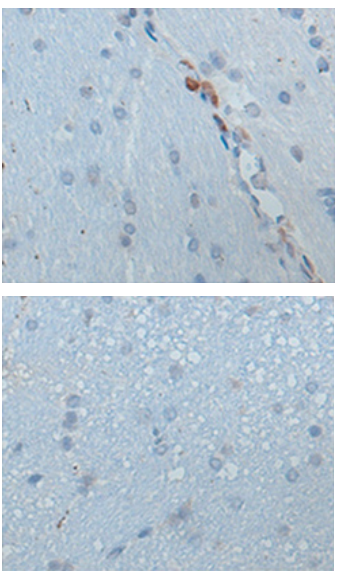

TBI
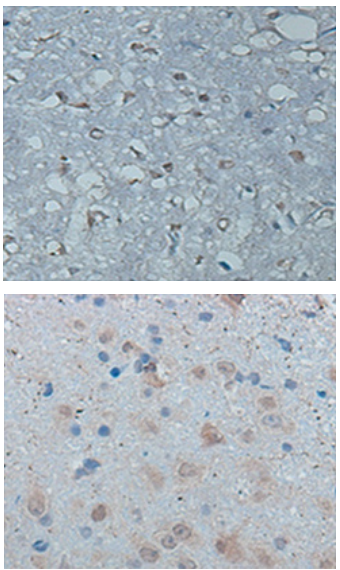

BMSC
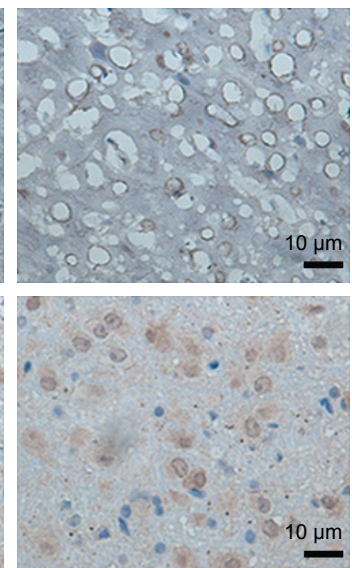

Figure 3 BMSCs transplantation attenuated neuronal apoptosis and diminished caspase-3 activation.

Notes: (A) TUNEL staining was performed to evaluate the apoptosis of brain neurons and (B) immunohistochemical staining was applied to observe the expression of caspase-3. Scale bar $=10 \mu \mathrm{m}$, magnification $\times 100$.

Abbreviations: BMSC, bone mesenchymal stem cell; TBI, traumatic brain injury; TUNEL, terminal deoxyribonucleotidyl transferase (TDT)-mediated dUTP-digoxigenin nick end labeling. 

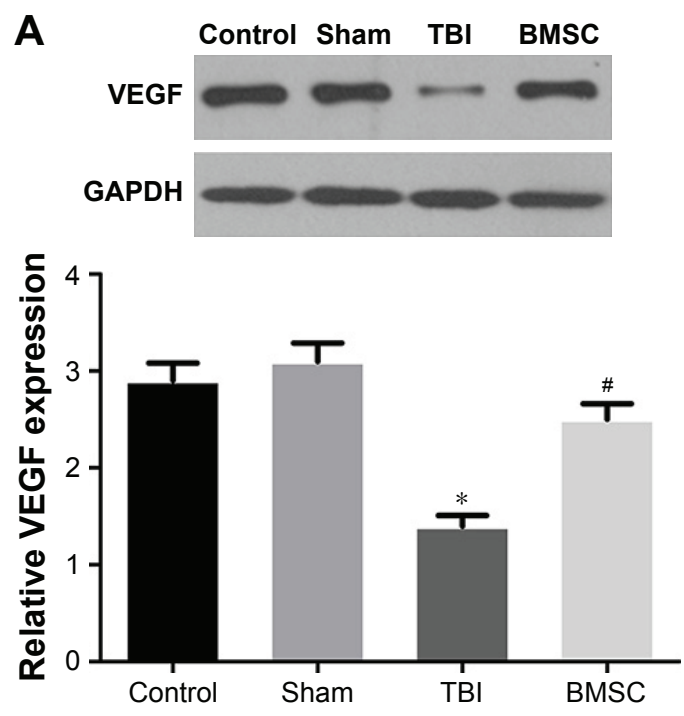
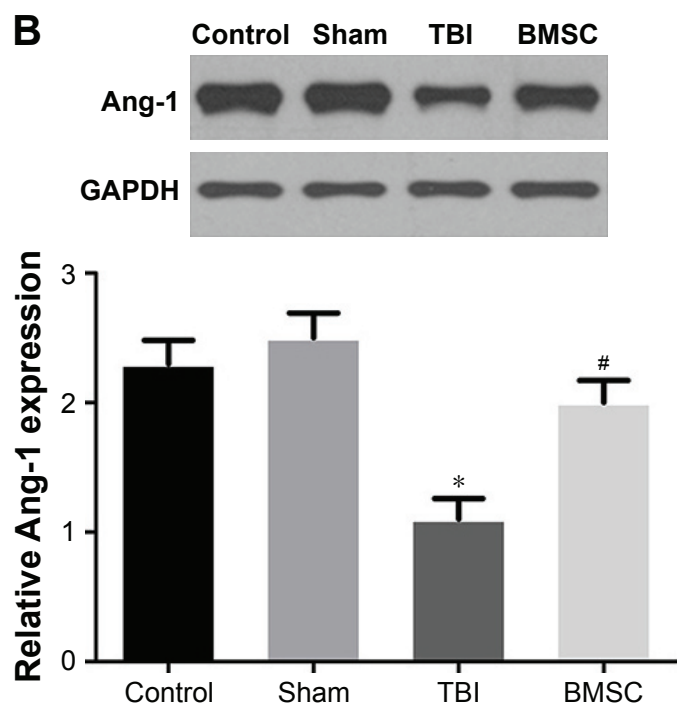

Figure 4 BMSCs transplantation upregulated expression of VEGF and Ang-I in brain tissues of TBI mice. The protein and mRNA levels of (A) VEGF and (B) Ang-I were detected by Western blot and qRT-PCR, respectively. GAPDH served as the control. ${ }^{*} P<0.05$ : Sham vs TBI, ${ }^{*} P<0.05$ : BMSC vs TBI.

Abbreviations: BMSC, bone mesenchymal stem cell; TBI, traumatic brain injury; VEGF, vascular endothelial growth factor; Ang-I, angiogenin-I; qRT-PCR, quantitative real-time polymerase chain reaction; GAPDH, glyceraldehyde-3-phosphate dehydrogenase.

than TBI mice (Figure 5A). Furthermore, MVD determined by CD34 was calculated and showed no significant difference between control and sham groups. MVD in the TBI group was markedly lower than in the control or sham group. BMSC group displayed higher MVD than TBI group but a little lower MVD than control and sham groups (Figure 5B). These results indicated that BMSCs transplantation promoted the formation of microvessels in brain tissues after TBI.

\section{Discussion}

TBI presents as a significant health concern and an enormous socioeconomic burden. Unfortunately, to date, there are no pharmacologic agents demonstrated to improve TBI outcomes. ${ }^{4}$ Thus, there is a compelling need to develop treatments for TBI. The safety and feasibility of BMSCs transplantation was confirmed in animal models ${ }^{24,25}$ and human trials. ${ }^{26,27}$ Our study found that cell therapies using BMSCs
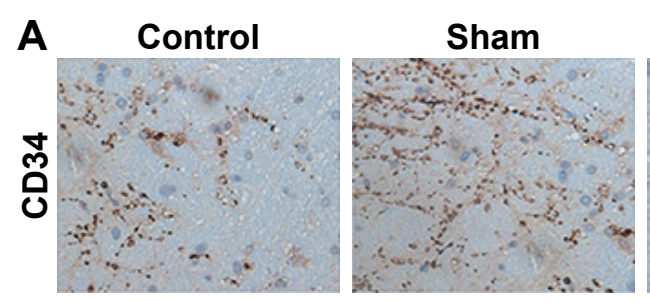

TBI

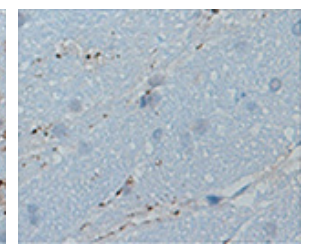

BMSC

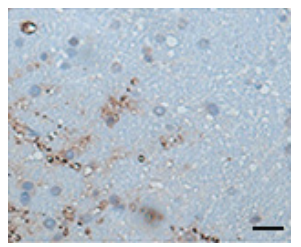

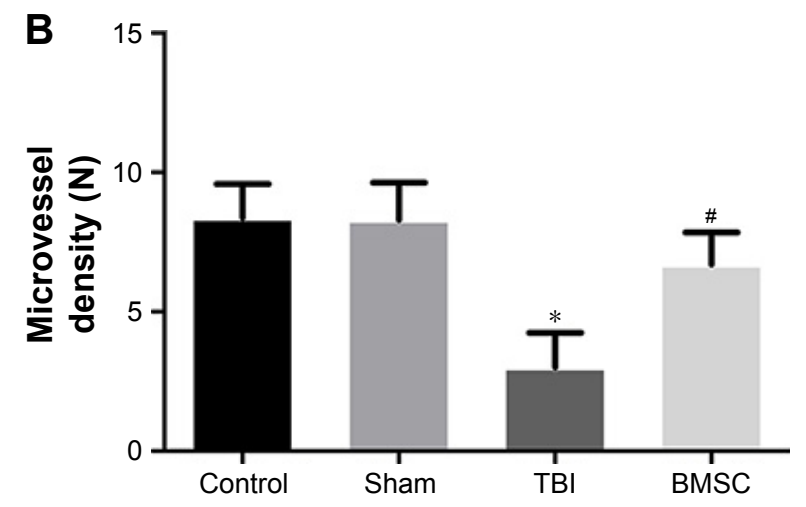

Figure 5 BMSCs transplantation promoted the formation of microvessels in brain tissues after TBI. (A) Microvessels stained by CD34 in brain tissues from mice in the four groups. Scale bar $=10 \mu \mathrm{m}$, magnification $\times 100$ and (B) MVD determined by CD34-positive microvessels in the four groups. ${ }^{* P}<0.05$ : Sham vs TBI, ${ }^{\# P<0.05: ~ B M S C ~ v s ~ T B I . ~}$ Abbreviations: BMSC, bone mesenchymal stem cell; TBI, traumatic brain injury; MVD, microvessel density; N, number. 
transplantation intravenously promoted angiogenesis and improved neurological function in TBI mice, thus provided supports for the treatment of TBI.

Misra et $\mathrm{al}^{28}$ indicated that the stem cell therapies are an emerging paradigm in strokes. Brenneman et $\mathrm{al}^{29}$ indicated that autologous bone marrow mononuclear cells can be safely harvested from rodents after stroke, and they are documented to migrate to the peri-infarct area, enhance recovery, and modulate the post-ischemic inflammatory response. Yang et $\mathrm{al}^{30}$ has well characterized the dose response, therapeutic time window, and biodistribiton of mononuclear cells from bone marrow in animal stroke models. Sharma et $\mathrm{al}^{31}$ demonstrated the potential of the intrathecal transplantation of autologous bone marrow mononuclear cells in improving the prognosis of functional recovery in chronic stage of stroke.

Bakhtiary et $\mathrm{al}^{32}$ stated that bromodeoxyuridine (Brdu)labeled BMSCs were present in the lesion boundary zone by immunohistochemistry analysis after intravenous injection of BMSCs in TBI rats, indicating BMSCs can survive, migrate, and differentiate into neurons in vivo. Also, Chen et $\mathrm{al}^{33}$ proposed that intraventricular injection of BMSCs increased the brain concentration of nerve growth factor and promoted neurological functional recovery in TBI mice. In accordance with this, in our study, we found that BMSCs transplantation by intravenous injection promoted recovery of neurological function in TBI mice, providing new evidence for the improvement of neurological function by BMSCs.

The cognitive dysfunction which TBI patients suffer from is mainly reflected by the impairment of learning and memory, attention, language and communication skills, the ability of identifying time and space, as well as logical reasoning. Mahmood et $\mathrm{al}^{34}$ reported that TBI rats treated with combination therapy of BMSCs and atorvastatin showed attenuated dysfunction of learning and memory reflected by the MWM test. Consistent with this, our study revealed that, compared to the TBI group, a reduction in escape latency and an increase in target zone entries, time in target zone, and number of times crossing the site were observed in the BMSC group, indicating intravenous transplantation of BMSCs effectively improved the learning and memory of TBI mice. In addition, our study also found that BMSCs transplantation ameliorated neuronal apoptosis and diminished activation of caspase-3, an important apoptotic executor in apoptotic cascades. Collectively, we can conclude that BMSCs improve neurological function in TBI mice.

Recent animal study has linked enhanced angiogenesis to improved performance in neurological and behavioral tests. ${ }^{35}$
Angiogenesis, the growth of new blood vessels, can provide tissue with oxygen and nutrition, ameliorate tissue ischemia, and promote the structural remodeling of damaged brain tissue, thus accelerating the repair of nerve function. Promoting angiogenesis may be a new approach to treat ischemic brain after TBI. ${ }^{4,36-39}$ Angiogenesis is regulated by the expression of various vascular growth factors and modulators, the most widely expressed and critical regulator of which is VEGF. This protein is secreted by tissues in response to ischemic and inflammatory stimuli and leads to endothelial migration, proliferation, and increased vascular permeability. ${ }^{40}$ In this study, levels of VEGF in brain gradually reduced 14 days after TBI, and similar results were observed with Ang-1, another stimulator of angiogenesis. It is noteworthy that BMSCs transplantation upregulated expressions of VEGF and Ang-1 to levels close to those found in control or sham group. These findings suggested that BMSCs transplantation may promote angiogenesis through upregulating VEGF and Ang-1, which was then confirmed by the formation of microvessels using immunohistochemistry and microvessel counting. Current investigations of the changes in molecular expression are increasing our understanding of the important role of BMSCs transplantation in TBI therapies; however, clinical studies should be performed to further validate our results. Molecular imaging has been emerging as a novel technology for visualizing metabolism and signal transduction to gene expression, ${ }^{41}$ which could be used in our further study to explore the signal transduction to VEGF and Ang-1 expression. In conclusion, our study demonstrated the important role of BMSCs transplantation in TBI mouse and indicated that the underlying mechanism was through promoting angiogenesis and improving neurological function. This provides a novel and effective strategy for cell-based therapy in the treatment of TBI.

\section{Disclosure}

The authors report no conflicts of interest in this work.

\section{References}

1. Xiong Y, Mahmood A, Chopp M. Animal models of traumatic brain injury. Nat Rev Neurosci. 2013;14(2):128-142.

2. Menon DK, Schwab K, Wright DW, Maas AI. Position statement: definition of traumatic brain injury. Arch Phys Med Rehabil. 2010;91(11): 1637-1640.

3. Walker PA, Shah SK, Harting MT, Cox CS Jr. Progenitor cell therapies for traumatic brain injury: barriers and opportunities in translation. Dis Model Mech. 2009;2(1-2):23-38.

4. Xiong Y, Zhang Y, Mahmood A, Chopp M. Investigational agents for treatment of traumatic brain injury. Expert Opin Investig Drugs. 2015 24(6):743-760

5. Maas AI, Stocchetti N, Bullock R. Moderate and severe traumatic brain injury in adults. Lancet Neurol. 2008;7(8):728-741. 
6. Xu B, Gao Y, Zhan S, et al. Quantitative protein profiling of hippocampus during human aging. Neurobiol Aging. 2016;39:46-56.

7. Rosenfeld JV, Maas AI, Bragge P, Morganti-Kossmann MC, Manley GT, Gruen RL. Early management of severe traumatic brain injury. Lancet. 2012;380(9847):1088-1098.

8. James S, Fox J, Afsari F, et al. Multiparameter analysis of human bone marrow stromal cells identifies distinct immunomodulatory and differentiation-competent subtypes. Stem Cell Reports. 2015;4(6): 1004-1015.

9. Bao C, Wang Y, Min H, et al. Combination of ginsenoside Rg1 and bone marrow mesenchymal stem cell transplantation in the treatment of cerebral ischemia reperfusion injury in rats. Cell Physiol Biochem. 2015;37(3):901-910.

10. Parekkadan B, Milwid JM. Mesenchymal stem cells as therapeutics. Annu Rev Biomed Eng. 2010;12:87-117.

11. Malgieri A, Kantzari E, Patrizi MP, Gambardella S. Bone marrow and umbilical cord blood human mesenchymal stem cells: state of the art. Int J Clin Exp Medicine. 2010;3(4):248-269.

12. Tse WT, Pendleton JD, Beyer WM, Egalka MC, Guinan EC. Suppression of allogeneic T-cell proliferation by human marrow stromal cells: implications in transplantation. Transplantation. 2003;75(3):389-397.

13. Fouillard L, Chapel A, Bories D, et al. Infusion of allogeneic-related HLA mismatched mesenchymal stem cells for the treatment of incomplete engraftment following autologous haematopoietic stem cell transplantation. Leukemia. 2007;21(3):568-570.

14. Nandoe Tewarie RD, Hurtado A, Levi AD, Grotenhuis JA, Oudega M. Bone marrow stromal cells for repair of the spinal cord: towards clinical application. Cell Transplant. 2006;15(7):563-577.

15. Yalvac ME, Yilmaz A, Mercan D, et al. Differentiation and neuroprotective properties of immortalized human tooth germ stem cells. Neurochem Res. 2011;36(12):2227-2235.

16. Wei L, Fraser JL, Lu ZY, Hu X, Yu SP. Transplantation of hypoxia preconditioned bone marrow mesenchymal stem cells enhances angiogenesis and neurogenesis after cerebral ischemia in rats. Neurobiol Dis. 2012;46(3):635-645.

17. Hunt RF, Scheff SW, Smith BN. Synaptic reorganization of inhibitory hilar interneuron circuitry after traumatic brain injury in mice. J Neurosci. 2011;31(18):6880-6890.

18. Leinhase I, Rozanski M, Harhausen D, et al. Inhibition of the alternative complement activation pathway in traumatic brain injury by a monoclonal anti-factor B antibody: a randomized placebo-controlled study in mice. $J$ Neuroinflammation. 2007;4:13.

19. Stahel PF, Shohami E, Younis FM, et al. Experimental closed head injury: analysis of neurological outcome, blood-brain barrier dysfunction, intracranial neutrophil infiltration, and neuronal cell death in mice deficient in genes for pro-inflammatory cytokines. J Cereb Blood Flow Metab. 2000;20(2):369-380.

20. Inostroza M, Cid E, Brotons-Mas J, et al. Hippocampal-dependent spatial memory in the water maze is preserved in an experimental model of temporal lobe epilepsy in rats. PloS one. 2011;6(7):e22372.

21. Sun YX, Dai DK, Liu R, et al. Therapeutic effect of SN50, an inhibitor of nuclear factor-kappaB, in treatment of TBI in mice. Neurol Sci. 2013; 34(3):345-355.

22. Ding S, Li C, Lin S, et al. Comparative evaluation of microvessel density determined by CD34 or CD105 in benign and malignant gastric lesions. Hum Pathol. 2006;37(7):861-866.

23. Weidner N, Semple JP, Welch WR, Folkman J. Tumor angiogenesis and metastasis - correlation in invasive breast carcinoma. $N$ Eng J Med. 1991;324(1):1-8.

24. Lu D, Mahmood A, Wang L, Li Y, Lu M, Chopp M. Adult bone marrow stromal cells administered intravenously to rats after traumatic brain injury migrate into brain and improve neurological outcome. Neuroreport. 2001;12(3):559-563.
25. Mahmood A, Lu D, Qu C, Goussev A, Chopp M. Human marrow stromal cell treatment provides long-lasting benefit after traumatic brain injury in rats. Neurosurgery. 2005;57(5):1026-1031; discussion 1026-1031.

26. Zhang ZX, Guan LX, Zhang K, Zhang Q, Dai LJ. A combined procedure to deliver autologous mesenchymal stromal cells to patients with traumatic brain injury. Cytotherapy. 2008;10(2):134-139.

27. Tian $C$, Wang $X$, Wang $X$, et al. Autologous bone marrow mesenchymal stem cell therapy in the subacute stage of traumatic brain injury by lumbar puncture. Exp Clin Transplant. 2013;11(2):176-181.

28. Misra V, Yang B, Sharma S, Savitz S. Cell-Based Therapy for Stroke. Totowa, NJ: Humana Press; 2011:143-161.

29. Brenneman M, Sharma S, Harting M, et al. Autologous bone marrow mononuclear cells enhance recovery after acute ischemic stroke in young and middle-aged rats. $J$ Cereb Blood Flow Metab. 2010;30(1): 140-149.

30. Yang B, Strong R, Sharma S, et al. Therapeutic time window and dose response of autologous bone marrow mononuclear cells for ischemic stroke. J Neurosci Res. 2011;89(6):833-839.

31. Sharma A, Sane H, Gokulchandran N, et al. Autologous bone marrow mononuclear cells intrathecal transplantation in chronic stroke. Stroke Res Treat. 2014;2014(2014):234095.

32. Bakhtiary M, Marzban M, Mehdizadeh M, et al. Comparison of transplantation of bone marrow stromal cells (BMSC) and stem cell mobilization by granulocyte colony stimulating factor after traumatic brain injury in rat. Iran Biomed J. 2010;14(4):142-149.

33. Chen Q, Long Y, Yuan X, et al. Protective effects of bone marrow stromal cell transplantation in injured rodent brain: synthesis of neurotrophic factors. J Neurosci Res. 2005;80(5):611-619.

34. Mahmood A, Lu D, Qu C, Goussev A, Chopp M. Treatment of traumatic brain injury with a combination therapy of marrow stromal cells and atorvastatin in rats. Neurosurgery. 2007;60(3):546-553; discussion 553-554.

35. Caplan AI, Dennis JE. Mesenchymal stem cells as trophic mediators. J Cell Biochem. 2006;98(5):1076-1084.

36. Xiong Y, Qu C, Mahmood A, et al. Delayed transplantation of human marrow stromal cell-seeded scaffolds increases transcallosal neural fiber length, angiogenesis, and hippocampal neuronal survival and improves functional outcome after traumatic brain injury in rats. Brain Res. 2009;1263:183-191.

37. Xiong Y, Mahmood A, Meng Y, et al. Delayed administration of erythropoietin reducing hippocampal cell loss, enhancing angiogenesis and neurogenesis, and improving functional outcome following traumatic brain injury in rats: comparison of treatment with single and triple dose. J Neurosurg. 2010;113(3):598-608.

38. Lu D, Goussev A, Chen J, et al. Atorvastatin reduces neurological deficit and increases synaptogenesis, angiogenesis, and neuronal survival in rats subjected to traumatic brain injury. J Neurotrauma. 2004; 21(1):21-32.

39. Xiong Y, Mahmood A, Chopp M. Angiogenesis, neurogenesis and brain recovery of function following injury. Curr Opin Investig Drugs. 2010;11(3):298-308.

40. Pandya NM, Dhalla NS, Santani DD. Angiogenesis - a new target for future therapy. Vascul Pharmacol. 2006;44(5):265-274.

41. Sandu N, Schaller B. Stem cell transplantation in brain tumors: a new field for molecular imaging? Mol Med. 2010;16(9):433-437. 
Neuropsychiatric Disease and Treatment

Dovepress

\section{Publish your work in this journal}

Neuropsychiatric Disease and Treatment is an international, peerreviewed journal of clinical therapeutics and pharmacology focusing on concise rapid reporting of clinical or pre-clinical studies on a range of neuropsychiatric and neurological disorders. This journal is indexed on PubMed Central, the 'PsycINFO' database and CAS,

and is the official journal of The International Neuropsychiatric Association (INA). The manuscript management system is completely online and includes a very quick and fair peer-review system, which is all easy to use. Visit http://www.dovepress.com/testimonials.php to read real quotes from published authors.

Submit your manuscript here: http://www.dovepress.com/neuropsychiatric-disease-and-treatment-journal 